\title{
USING MACHINE LEARNING TECHNIQUES FOR DATA QUALITY MONITORING AT CMS EXPERIMENT
}

\author{
GUILLERMO A. FIDALGO RODRÍGUEZ \\ PHYSICS DEPARTMENT \\ UNIVERSITY OF PUERTO RICO MAYAGÜEZ
}

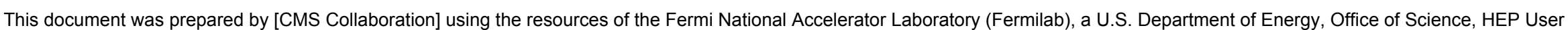
Facility. Fermilab is managed by Fermi Research Alliance, LLC (FRA), acting under Contract No. DE-AC02-07CH11359. 


\section{CMS DETECTOR}

Total weight

, 000 tonnes

Overall diameter : $15.0 \mathrm{~m}$

Overall length $\quad: 28.7 \mathrm{~m}$

Magnetic field

$: 3.8 \mathrm{~T}$

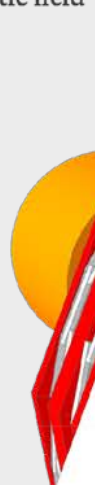

CRYSTAL

ELECTROMAGNETIC

CALORIMETER (ECAL)

$\sim 76,000$ scintillating $\mathrm{PbWO}_{4}$ crystals
STEEL RETURN YOKE 12,500 tonnes

\section{THE COMP ACT MUON SOLENOID (CMS) DETECTOR AT}

\section{LHC}

\section{SILICON TRACKERS}

Pixel $(100 \times 150 \mu \mathrm{m}) \sim 16 \mathrm{~m}^{2} \sim 66 \mathrm{M}$ channels

Microstrips $(80 \times 180 \mu \mathrm{m}) \sim 200 \mathrm{~m}^{2} \sim 9.6 \mathrm{M}$ channels

SUPERCONDUCTING SOLENOID Niobium titanium coil carrying $\sim 18,000 \mathrm{~A}$

HADRON CALORIMETER (HCAL)

Brass + Plastic scintillator $\sim 7,000$ channels

\section{MUON CHAMBERS}

Barrel: 250 Drift Tube, 480 Resistive Plate Chambers Endcaps: 468 Cathode Strip, 432 Resistive Plate Chambers

PRESHOWER

Silicon strips $\sim 16 \mathrm{~m}^{2} \sim 137,000$ channels

FORWARD CALORIMETER Steel + Quartz fibres $\sim 2,000$ Channels r
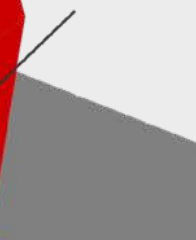


\section{OBJECTIVES}

- Apply recent progress in Machine Learning techniques regarding a utomation of DQM scrutiny for HCAL

- To focus on the Online DQM.

- To compare the performance of different MLa lgorithms.

- To compare fully supervised vs semi-supervised approach.

- Impact the current workflow, make it more efficient and can gua rantee that the data is use ful for physics a na lysis. 


\section{CHALLENGE}

- Make sure detector behaves well to perform sensible data analysis.

- Reduce man power to discriminate good and bad data, spot problems, save time examining hundreds of histograms.

- By building intelligence to analyze data, raise alarms, quick feedback.

- Implementing the best architecture for neural networks

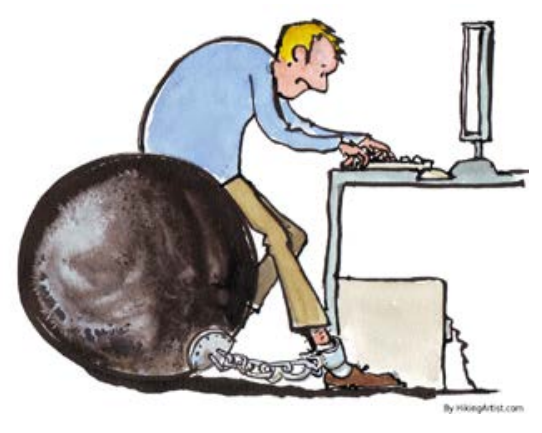

- Underfitting - Too simple and not able to learn

- Overfitting - Too complex and learns very specific and/or unnecessary features

- There is no rule of thumb

- Many, many, many......possible combinations. 


\section{WHAT IS DATA QUALITY MONITORING (DQM)?}

- Two kinds of workflows:

- Online DQM

- Provides feedback of live data taking.

- Alarms if something goes wrong.

- Offline DQM
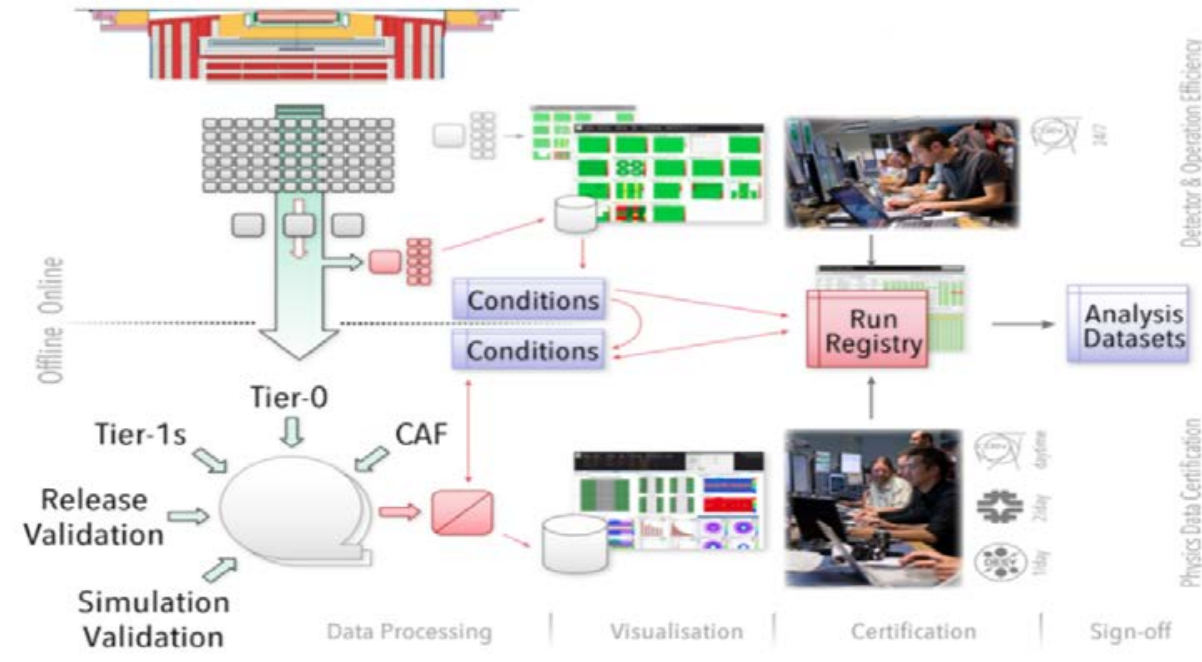

- After da ta taking

- Responsible for bookkeeping and certifying the finaldata with fine time granula rity. 


\section{HYPOTHESIS AND PROJECT QUERIES}

\section{Queries}

- Can we make an algorithm that identifies a noma lies in the data flow?

Hyp othe sis

- We can develop a ML a lgorithm that takes the images a s data and determine whether or not a n error is occurring.

Ra tiona le

- Since this a lgorithm takes images a s inputs it can learn to compare the images given with a baseline and correctly identify patterns and deviations from the baseline. 


\section{TOOLS AND DATA PROCESSING}

- Working env: python Jupyter notebook

\section{TensorFlow}

- Kera s (with Tensorflow as backend) and Scikitle arn

- Creation of a model

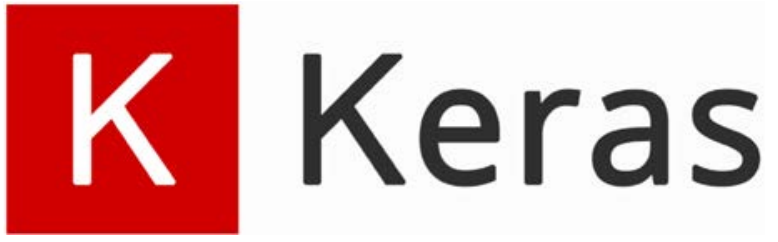

- Tra in and test its performance

- The input data consists of occupancy maps

- one map for each luminosity section

- Used 2017 good da ta and genera te bad da ta artificia lly 


\section{IMAGE ANALYSIS TERMINOLOGY}

- Hot - image with noisy (red)cha nnels

- Dead - image with inactive (blue)channels

- Good - regular images that a re certified for a nalysis

- Model - an ML a lgorithm's structure

- Loss - number that represents distance from target value 


\section{IMAGES AND READOUT CHANNELS USED AS INPUTS FOR THE ML ALGORITHM}

- Supervised and Semi-Supervised Learning

- 5x5 problematic region with random location

- 5x5 (readout channels) problematic region with fixed location

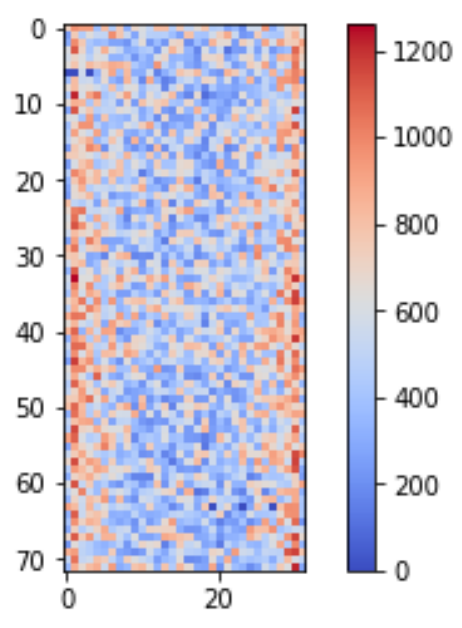

Good

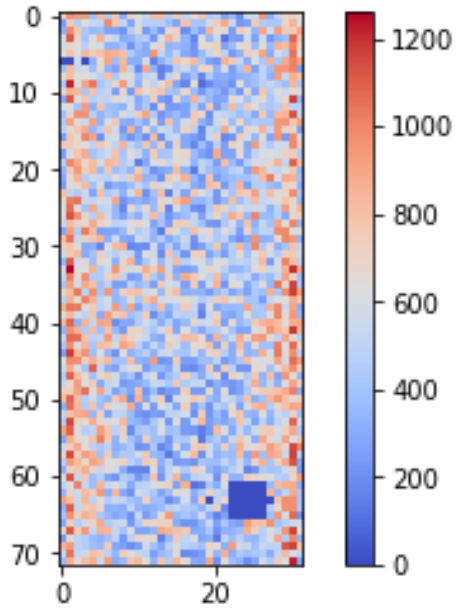

Dead

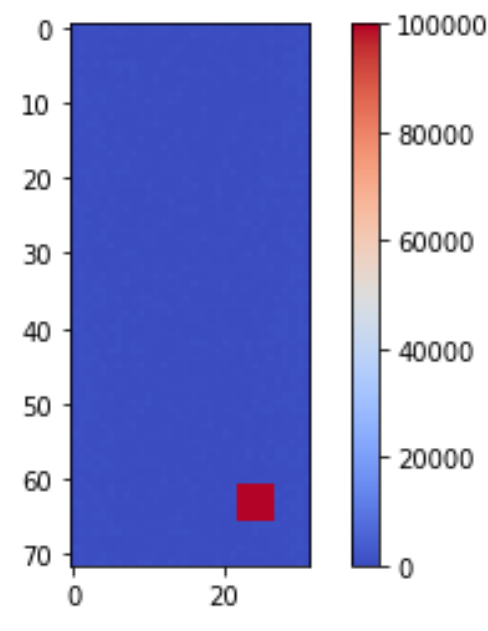

Hot 


\section{SUPERVISED LEARNING}

accuracy score: 0.950792326939
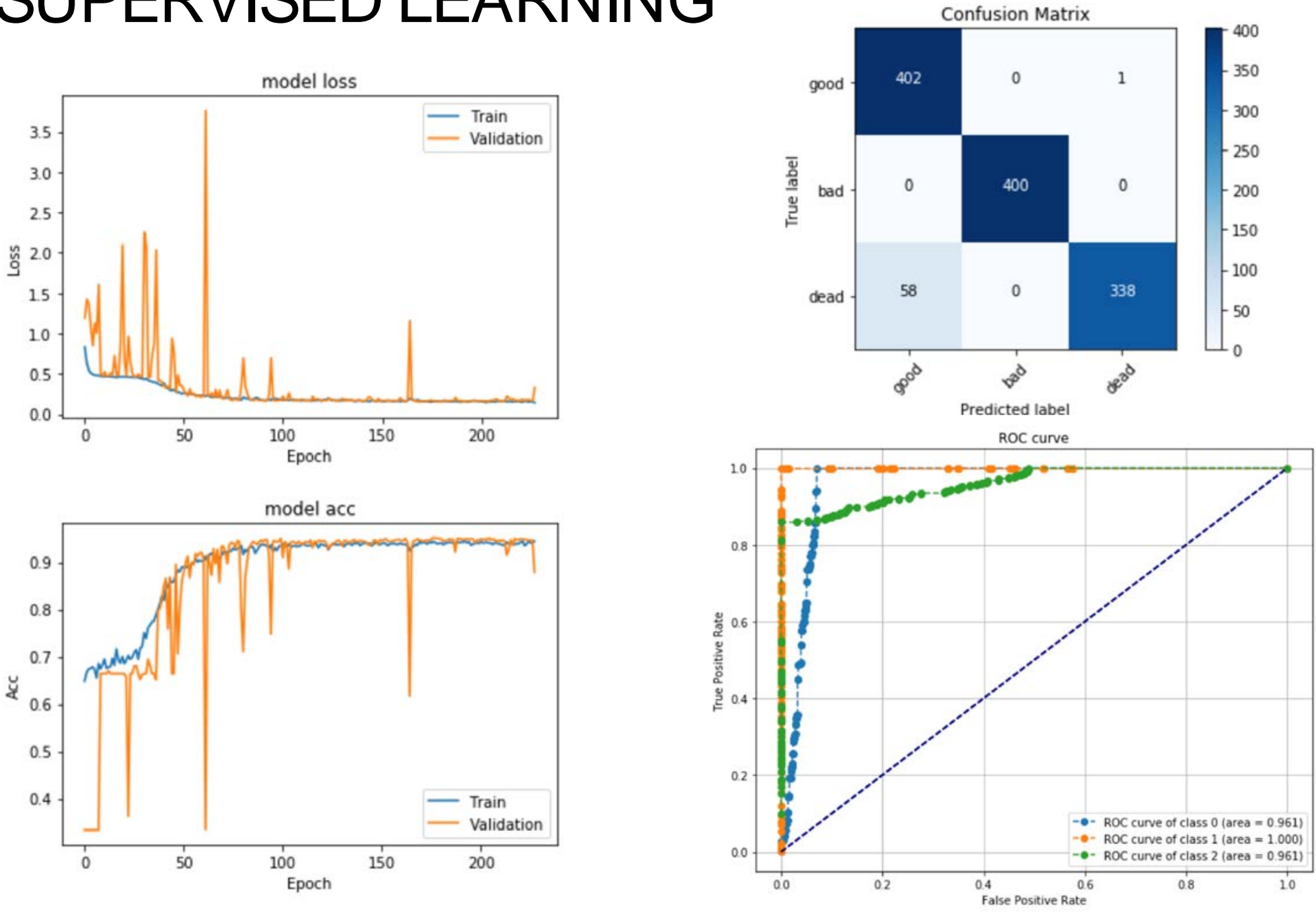

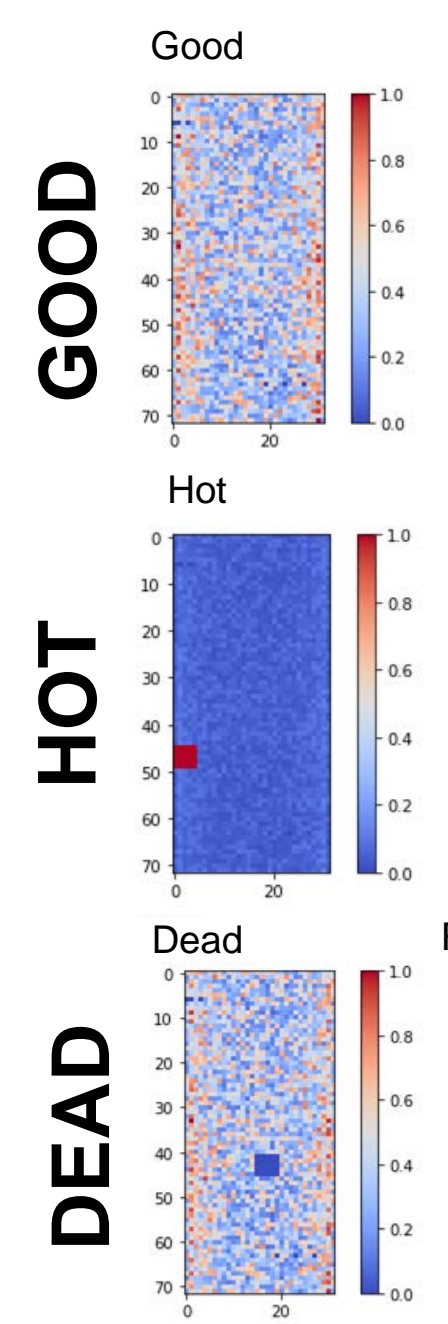

Reconstruction

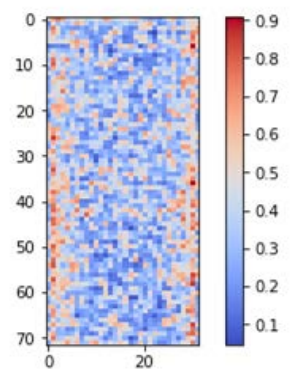

Reconstruction

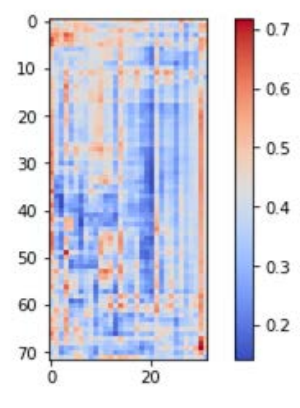

Reconstruction

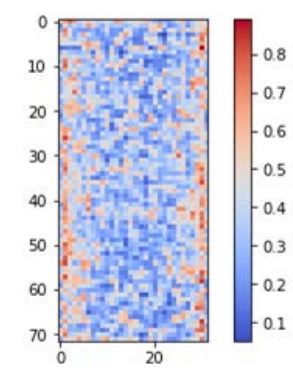

- Trained only on good images

- Expected to see better reconstruction for good images and a much different reconstruction for bad images.

- Bad images have $5 \times 5$ bad regions

- Hot

- Dead

- Images have been normalized

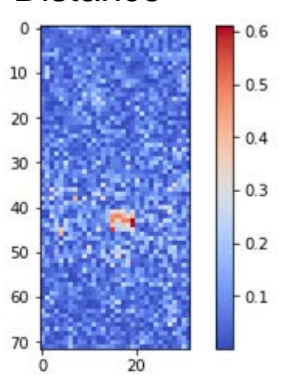

- this architecture seems to perform best for us. 


\section{ERROR DISTRIBUTION PER IMAGE CLASS}

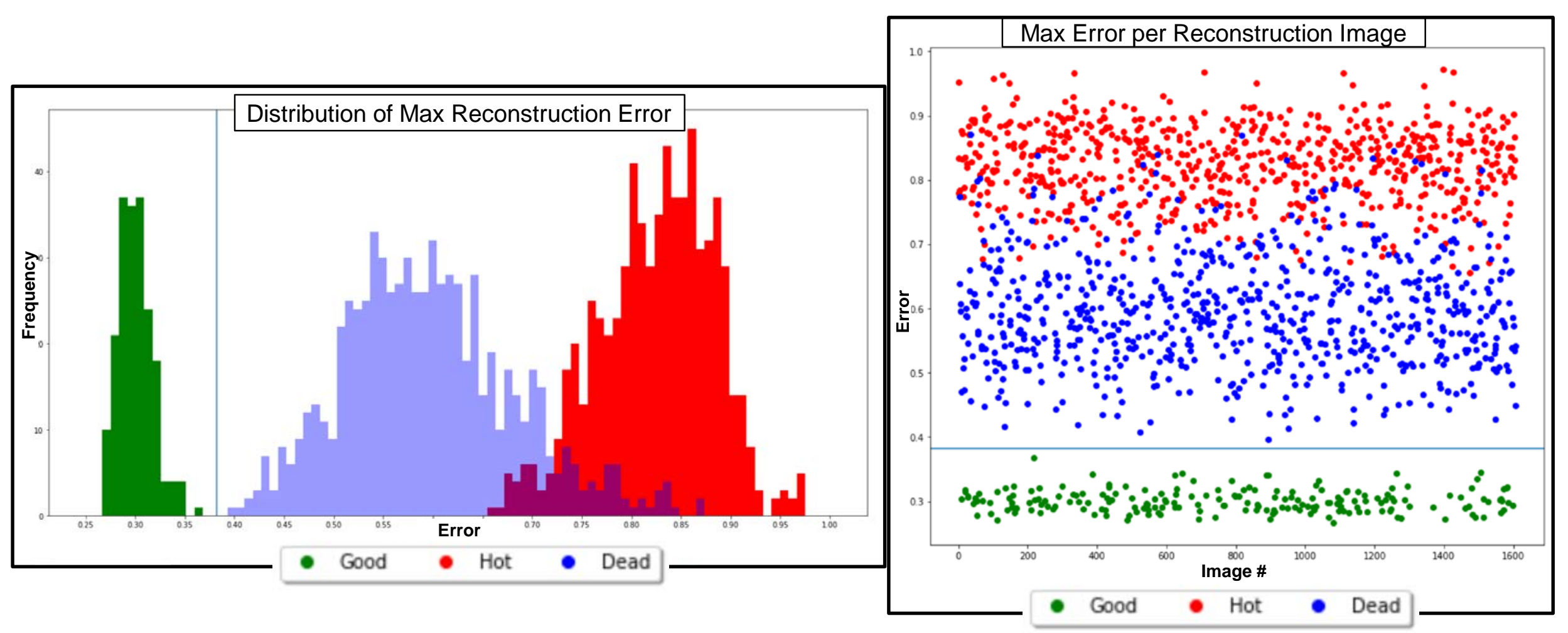




\section{WHAT'S NEXT?}

- Why a nd exactly what is it lea rning?

- Can we make it work with something more rea listic?

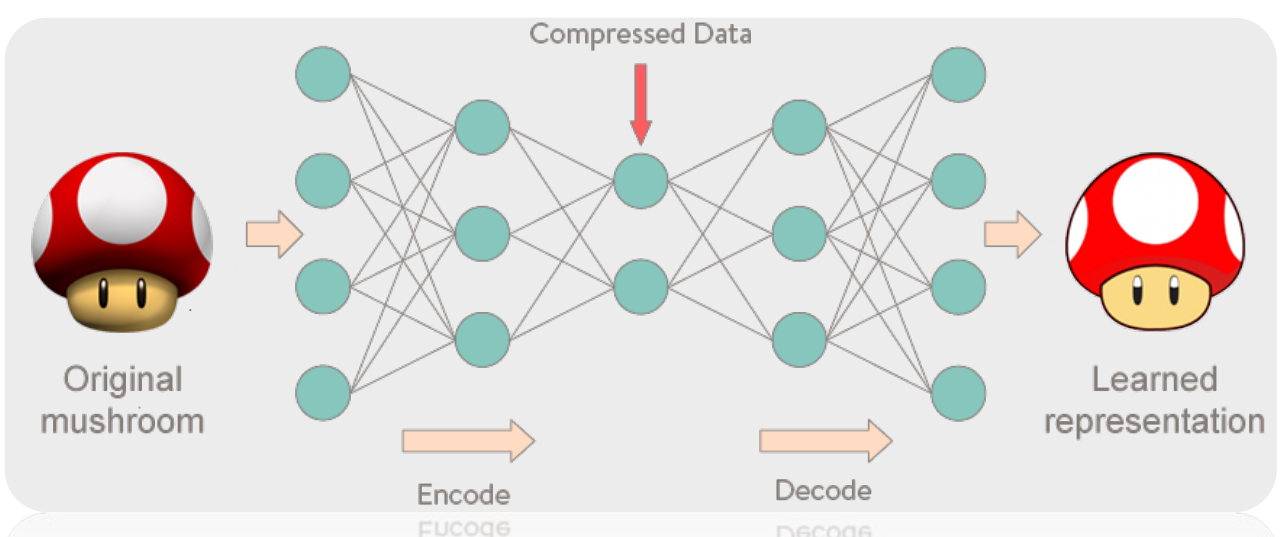

- 1x1 bad region (channel)

- Can it identify what va lues should be expected after each lumi-section?

- Move from artificialbad data to real cases of bad data (in progress)

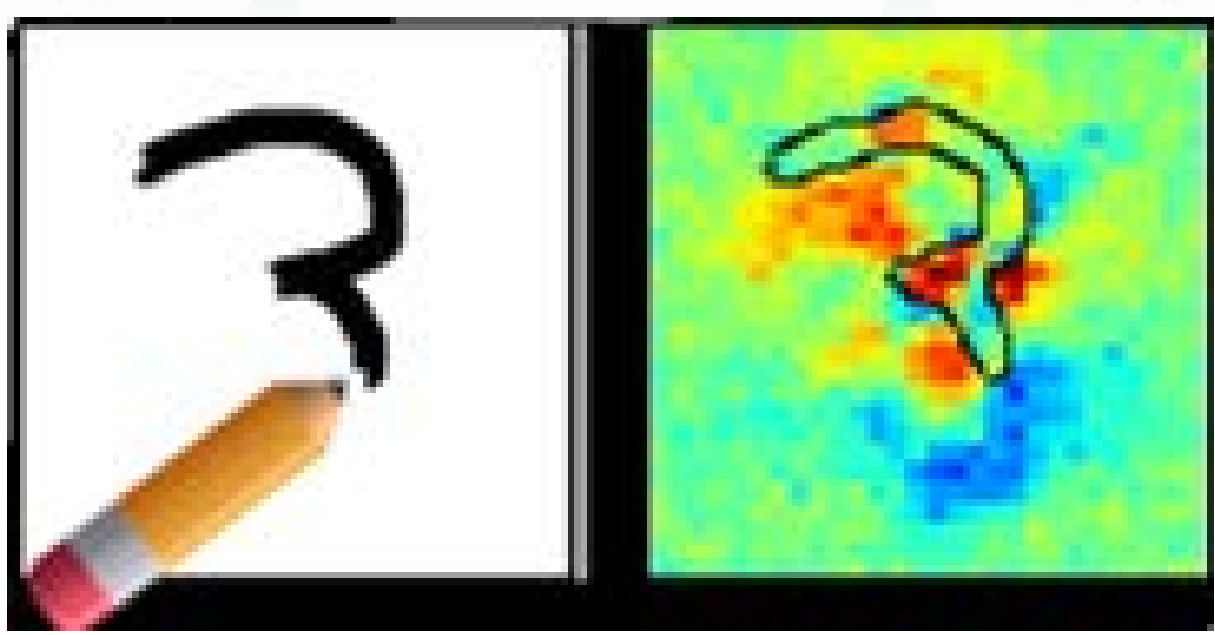




\section{Acknowledgments}

- The US State Dept.

- The University of Michiga $n$

- CERN/CMS

- Federic o De Guio , Ph.D (Texa s Tech)

- Nural Akchurin, Ph.D (Texa s Tech)

- Sudhir Ma lik, Ph.D (University of Puerto Rico Ma ya güez)

- Steven Gold farb, Ph.D (University of Melbourne)

- J ean Krisch, Ph.D (University of Michigan) 